\title{
Polymer, Additives, and Processing Effects on N95 Filter Performance
}

Gregory S. Larsen,* Yongqiang Cheng, Luke L. Daemen, Tej N. Lamichhane, Dale K. Hensley, Kunlun Hong, Harry M. Meyer, III, Steven J. Monaco, Alan M. Levine, Richard J. Lee, Emma Betters, Kim Sitzlar, Jesse Heineman, Justin West, Peter Lloyd, Vlastimil Kunc, Lonnie Love, Merlin Theodore,* and Mariappan Parans Paranthaman*

Cite This: https://dx.doi.org/10.1021/acsapm.0c01294

ABSTRACT: The current severe acute respiratory syndrome coronavirus 2 (SARS-COV-2) pandemic has highlighted the need for personal protective equipment, specifically filtering facepiece respirators like N95 masks. While it is common knowledge that polypropylene (PP) is the industry standard material for filtration media, trial and error is often required to identify suitable commercial precursors for filtration media production. This work aims to identify differences between several commercial grades of PP and demonstrate the development of N95 filtration media with the intent that the industry partners can pivot and help address N95 shortages. Three commercial grades of high
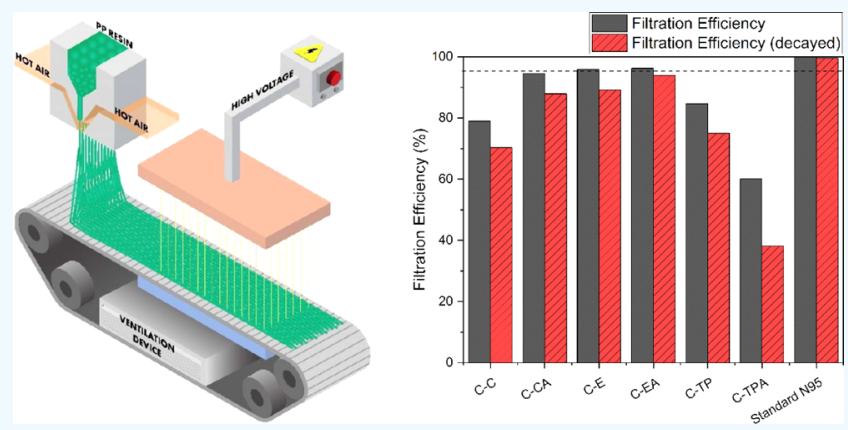
melt flow index PP were melt blown at Oak Ridge National Laboratory and broadly characterized by several methods including differential scanning calorimetry (DSC), X-ray diffraction (XRD), and neutron scattering. Despite the apparent similarities (high melt flow and isotacticity) between PP feedstocks, the application of corona charging and charge enhancing additives improve each material to widely varying degrees. From the analysis performed here, the most differentiating factor appears to be related to crystallization of the polymer and the resulting electret formation. Materials with higher crystallization onset temperatures, slower crystallization rates, and larger number of crystallites form a stronger electret and are more effective at filtration.

KEYWORDS: isotactic polypropylene, melt blowing, N95, filtration, crystallization, electret

\section{INTRODUCTION}

The recent and ongoing severe acute respiratory syndrome coronavirus 2 (SARS-COV-2) pandemic has exposed the supply chain challenges within the medical field. Specifically, there is a shortage of filtration media and other personal protective equipment for healthcare workers. Prior work has investigated the cleaning methods of filtering facepiece respirators (FFRs $)^{1-3}$ to help address shortages during an epidemic or use of medical masks to strategically conserve true FFRs. ${ }^{4}$ Likewise, the large-scale vapor phase hydrogen peroxide (VPHP) decontamination method has been implemented $^{5}$ and approved for use. ${ }^{6}$ Even with decontamination capabilities and optimized use, the ability to manufacture quality N95 filtration media needs to expand to new and local manufacturing sites around the nation and world to address the increased demand. The standard most commonly sought respiratory protection is N95, a NIOSH (National Institute for Occupational Safety and Health) designation for a respirator that filters greater than $95 \%$ of nonoil containing $\sim 0.3 \mu \mathrm{m}$ size particles as defined in CFR
Title 42 Chapter I Subchapter G part 84 subpart K (other classifications exist such as N99 or N100 respirators capable of filtering 99 or $99.97 \%$ of particles, respectively, or P95 filters capable of filtering oil-containing aerosols). The specification defines inhalation and exhalation pressure drops (35 and 25 $\mathrm{mm} \mathrm{H}_{2} \mathrm{O}$, respectively), the use of sodium chloride aerosols to test particle penetration, test airflow rates, etc. Filtration systems may be tested using ASTM F2100 and ASTM F2299 standards. To ensure suitable performance over the shelf life of the product, commercial N95 filters are overdesigned and initially filter more than the required $95 \%$ such that after any decay, the filters still meet or exceed the specified performance. N95 respirators are typically made of three plies of material, a

Received: November 23, 2020

Accepted: January 11, 2021 
melt-blown polypropylene (PP) electret sandwiched between two layers of spun-bond polypropylene. Many polymers can be melt blown into nonwoven webs, but polypropylene (PP) is the most widely used due to its ease of processing and effectiveness as a filter medium. ${ }^{7}$ Additionally, PP is low density, hydrophobic, has good chemical stability, low melt temperatures for easy bonding, and good mechanical strength. The spun-bond layers (made by a process similar to melt blowing) provide mechanical strength, while the melt-blown layer provides all the filtration (and pressure drop) needed. The weight of the melt-blown fabric and the number of plies can be varied depending on the application (medical vs N95, etc.); however, N95 masks typically have two plies of 30-35 $\mathrm{g} / \mathrm{m}^{2}$ melt-blown fabrics. The application of corona charge to the melt-blown fibers vastly improves the filtration efficiency due to the formation of electrets.

To achieve the required fiber diameter and filter performance, PP is typically melt-blown. ${ }^{8,9}$ In the melt blowing process, as seen in Figure S1 (Supporting Information), a polymer is extruded through small-diameter holes $(0.2-1 \mathrm{~mm})$ with $\mathrm{L} / \mathrm{D}$ (length/diameter) ratios on the order of 10-30 and hole densities of 50-500 holes per centimeter. ${ }^{7,8,10}$ Hot air is blown on either side of the extrusion point and stretches the fibers as they are collected against a mesh conveyor belt. Fibers undergo extreme elongation (factors on the order of $250^{11}$ ) and cooling $\left(10^{3}{ }^{\circ} \mathrm{C} / \mathrm{s}\right) \cdot{ }^{12,13}$ Filtration occurs by the following four major mechanisms: inertial impaction, interception, diffusion, and electrostatic interaction. ${ }^{14-16}$ The formation of smaller fibers as well as thicker and denser matts will improve all filtration characteristics, except electrostatic interaction, and generally increase the pressure drop..$^{10,17,18}$ An electret is an electric analog of a magnet and creates a permanent electric field. The formation of electrets in fibrous filter media creates the ability to significantly enhance filtration efficiency without increasing the pressure drop. The application of charge via corona treatment creates electrets within the fabric that greatly enhance the filtration efficiency. ${ }^{19-21}$ The charge storage capacity of electret is related to charge traps that prohibit or restrain charge drift through surface and/or bulk. ${ }^{22-24}$ In PP, the charge traps occur at crystalline boundaries; nucleating agents that increase the number of spherulites and crystalline boundaries will increase the charge storage capacity. ${ }^{25}$ About 0.5 wt \% stearate added to PP was melt-blown and shown to improve the filtration efficiency initially, the stability over time, and at elevated temperatures (over $70{ }^{\circ} \mathrm{C}$, up to $110{ }^{\circ} \mathrm{C}$ ). ${ }^{25}$ Substituted benzene-1,3,5-tricarboxylic acid trisamides are effective nucleating agents for isostatic polypropylene (iPP) and have been shown to improve the electret properties in films, particularly in combination with processing conditions that create isolated aggregates that act as efficient charge traps. $^{26}$ Here, we report the melt blowing of three commercially available isotactic polypropylenes into filtration media. The effect of corona treatment and the inclusion of a charge enhancing additive on overall filtration efficiency are determined. The resulting materials are analyzed by a wide range of techniques including differential scanning calorimetry (DSC), X-ray diffraction (XRD), and neutron scattering to identify the key attributes of the materials for making effective filtration media.

\section{MATERIALS AND MELT BLOWING OF FILTRATION MATERIAL}

2.1. Polymer Feedstocks. Three (3) grades of polypropylene were investigated. One material was Ziegler-Natta (ZN)-catalyzed isotactic PP and is a "vis-broken" polymer that undergoes chain scission during processing to vastly increase the melt flow rate (Total polymers 3926). The other two materials (Exxon PP6936G2 and a proprietary material used by our industry partner Cummins) were both metallocene-catalyzed isotactic PP with high melt flow rates. The materials will be referred to as TP, E, and $\mathrm{C}$, respectively. The polymerization methods, $\mathrm{ZN}$ and metallocene, produce iPP with slight variation. $\mathrm{ZN}$-catalyzed iPP do not have uniformly distributed irregularities, ${ }^{27}$ rather they have highly isotactic blocks, defect-rich segments, and syndiotactic segments. ${ }^{28}$ Isotactic ZN PP can be considered a mixture of different chains, and most commercial $\mathrm{ZN}$ iPP contain short atactic chains. ${ }^{29}$ Metallocene-catalyzed PP stereo defects are dependent on the catalyst symmetry, ${ }^{28}$ and their distribution is homogeneous. ${ }^{29}$ Metallocene-catalyzed iPP has a narrower molecular weight distribution than $\mathrm{ZN}$-catalyzed systems. Metallocene-synthesized iPP have shorter average isostatic sequences than ZN PPs with the same average stereo defects. ${ }^{30}$ The polymers were blended with a masterbatched additive with the goal of improving electret formation and ultimately filtration performance. The additive is a stearate in a high melt flow PP, supplied as pellets (Techmer PM: PPM13774) and requiring only physical blending in the melt blowing extruder. The filter media was melt-blown at ORNL's carbon fiber technology facility (CFTF) into the fabric of approximately 30 grams per square meter $\left(\mathrm{g} / \mathrm{m}^{2}\right)$. Multiple plies can then be combined to achieve the desired efficiency.

2.2. Melt Blowing Process and Corona Treatment. In all cases, the polymers were extruded through the same die of approximately five holes per centimeter and $0.2-0.5 \mathrm{~mm}$ diameter. The die to collector distance (DCD) was $40-60 \mathrm{~cm}$. The mesh collector belt was run at approximately $5-7 \mathrm{~m} / \mathrm{min}$ and balanced with the extrusion rate to create materials of approximately $30 \mathrm{~g} / \mathrm{m}^{2}$. The process was optimized for each material resulting in slight variations of the listed parameters. The photos of the feedstock materials and the melt-blown process including the video of the preparation of PP fabrics are shown in Figure S2. The melt-blown web passed through a proprietary corona treatment system capable of applying $30-60 \mathrm{kV}^{20}$ This electric field induces an electret, or permanent electric field, within the melt-blown fibers and thereby improve the filtration efficiency of the fabric while maintaining a low airflow resistance.

\section{RESULTS AND DISCUSSION}

3.1. Filtration Efficiency Determination. The filtration efficiency, $\mu$, is the fraction of particles that the filter stops. The penetration, $P$, is the fraction of particles that pass through the filter such that the sum of $\mu$ and $P$ is 1 . The use of multiple low basis weight plies, rather than a single high basis weight ply, constitutes the preferred manufacturing method as it allows for the production of lower basis weight fabrics that can be easily charged and reduces the likelihood of holes or low areal weight spots that span the entire filter thickness. In addition to filtration efficiency, the pressure drop across the filter helps determine the comfort and energy efficiency of the filter. While the $\mathrm{N} 95$ specification allows for $25-35 \mathrm{~mm} \mathrm{H}_{2} \mathrm{O}$ pressure drop, a comfortable mask should have a pressure drop of less than $10 \mathrm{~mm} \mathrm{H}_{2} \mathrm{O}$ and preferably less than $8 \mathrm{~mm} \mathrm{H}_{2} \mathrm{O}$. The results of the filtration efficiency for two plies of the $30 \mathrm{~g} / \mathrm{m}^{2}$ filtration media are summarized in Table 1 and Figure 1 . To make commercial grade filtration media, a manufacturer would further need to optimize the basis weight and number of plies. The feedstock materials are samples E, C, and TP; the materials can be modified by the addition of the additive as indicated by the suffix $A$; the melt-blown fabrics can be 
Table 1. Initial Filtration Efficiency for Two Plies of Filter Media Tested at 50 Liters per Minute (lpm) Flow Rate

\begin{tabular}{lcc}
\multicolumn{1}{c}{ sample } & restriction $\left(\mathrm{mm} \mathrm{H}_{2} \mathrm{O}\right)$ & efficiency $(\%)$ \\
standard N95 & 8.3 & 99.40 \\
C-EA & 5.3 & 96.21 \\
C-E & 5.3 & 95.84 \\
C-CA & 4.8 & 94.50 \\
C-TP & 5.8 & 84.70 \\
C-C & 4.5 & 79.00 \\
C-TPA & 4.2 & 60.10 \\
NC-E & 5.7 & 42.30 \\
NC-TPA & 5.6 & 39.00 \\
NC-EA & 5.0 & 30.30 \\
NC-TP & 5.4 & 28.50 \\
NC-CA & 5.0 & 27.70 \\
NC-C & 3.8 & 24.80 \\
\hline
\end{tabular}

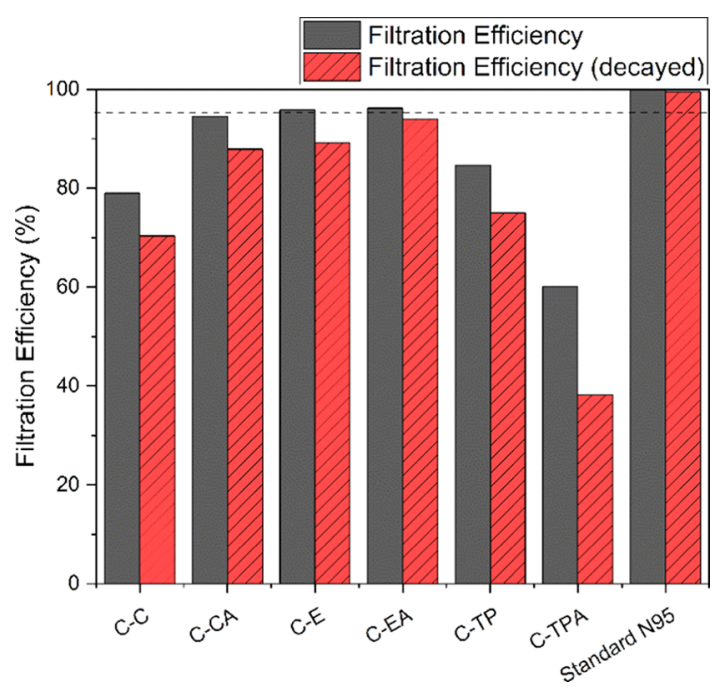

Figure 1. Filtration efficiency comparison for two plies of the material before and after aging for $24 \mathrm{~h}$ at $70{ }^{\circ} \mathrm{C}$ and $85 \% \mathrm{RH}$. The nucleating agent additive generally decreases the filtration efficiency decay. The dashed line represents $95 \%$ filtration efficiency.

charged or not charged, indicated by the prefix C- or NC-. Thus NC-EA is a noncharged fabric made from sample $\mathrm{E}$ with the additive. From Table 1, we clearly see that the application of charge to create an electret filter is critical in achieving high efficiency.

Comparing the filtration efficiencies in Table 1, sample E appears to produce the filter media with the highest filtration efficiencies in our process conditions. From the knowledge that increasing the crystalline-amorphous interfacial volume fraction within the sample will increase the number of charge traps to form an electret and therefore form a more efficient filter, the addition of a nucleating agent is hypothesized to increase the filtration efficiency of all materials. Counterintuitively, the nucleating agent changes the filtration efficiency differently in the three polymers: it significantly improves sample C, has minimal effect on sample E, and a detrimental effect on sample TP. In addition to initial performance, filtration efficiency must be maintained over the anticipated shelf life of the filter media. As seen in Table 1, the formation of the electret vastly improves the filtration performance, and any degradation of the electret over time can reasonably be expected to decrease performance. To test performance decay, samples are aged for $24 \mathrm{~h}$ at $70{ }^{\circ} \mathrm{C}$ and $85 \% \mathrm{RH}$ (relative humidity). After conditioning, samples were tested for filtration efficiency with a flow rate of $50 \mathrm{lpm}$, reported efficiencies are taken as the average over the 15 min test from two test specimens, and the resistance are measured as well (Figures 1 and S3).

3.2. Scanning Electron Microscopy (SEM) Analysis. The SEM images of typical melt-blown fibers showing fiber size and shapes are shown in Figure 2 (additional SEM images

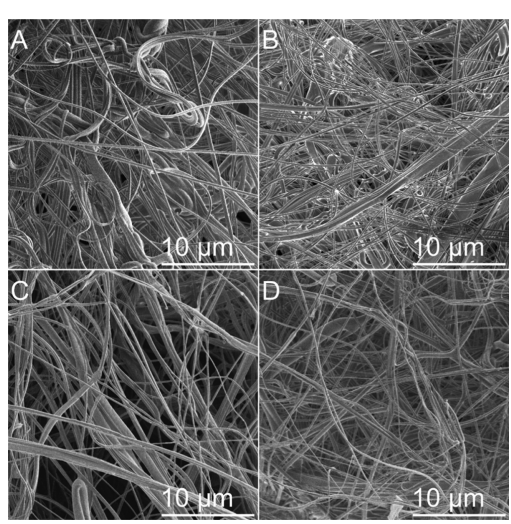

Figure 2. Representative SEM images of melt-blown fibers showing an enlarged version of larger-scale fiber arrangements. (A) Sample C charged with additive; (B) sample $\mathrm{E}$ charged with additive; (C) sample TP charged with additive; and (D) Standard N95 material. SEM images of other fibers are shown in the Supporting Information.

are shown in S4, S5, S6, S7, S8, S9, and S10). The SEM images were analyzed for fiber diameters. As shown in Figure 3, typical

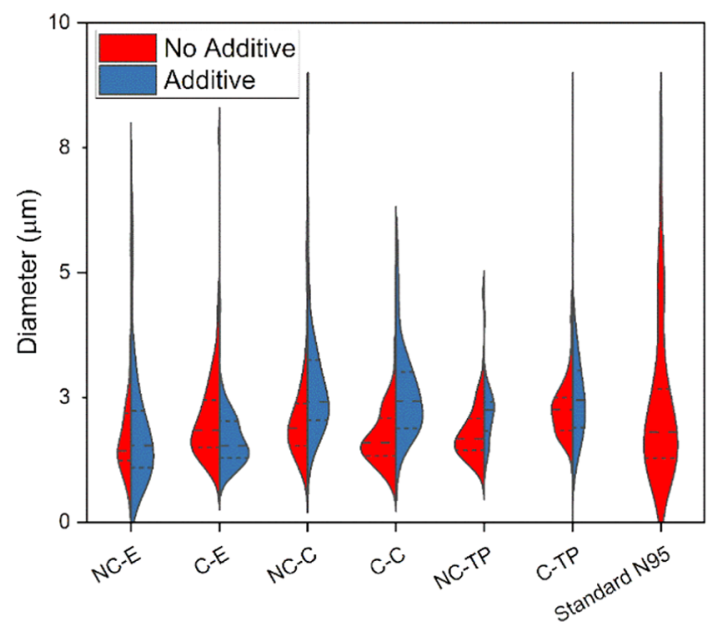

Figure 3. Violin plot showing the fiber diameter distribution for the three grades of polymer with and without applied charge split by the use of the additive as compared to standard N95 material.

fiber diameters range from 1 to $4 \mu \mathrm{m}$ in diameter with the average closer to $2 \mu \mathrm{m}$. The charged and noncharged samples were collected from the same melt blowing run; the only difference was the application of the corona treatment. The charged samples tend to have a higher fiber diameter and variability; the cause of this is not immediately clear.

3.3. Effect of Crystallinity, Crystallization Rates, and Crystallite Size. While DSC provides useful information on the degree and nature of crystallization, it is difficult to directly compare to the melt blowing process because melt blowing has 
faster cooling rates and additional strain-induced crystallization effects. Both isothermal and nonisothermal DSC were conducted to understand differences in crystallization onset and rate between polymer grades and blends. Further DSC analysis was conducted on melt-blown samples to determine the degree of crystallinity in various samples; this DSC crystallinity data is compared to XRD analysis.

Isothermal DSC gives the time to peak heat flow during crystallization. This shows differences in crystallization rates for the different materials under isothermal cooling conditions. Samples were heated to $200{ }^{\circ} \mathrm{C}$ and then cooled to either 105 , 110,115 , or $120{ }^{\circ} \mathrm{C}$ at the maximum cooling rate of the instrument, $\approx 65{ }^{\circ} \mathrm{C} / \mathrm{min}$. Considering the crystallization time as a function of isothermal cooling temperature in Figure 4, we

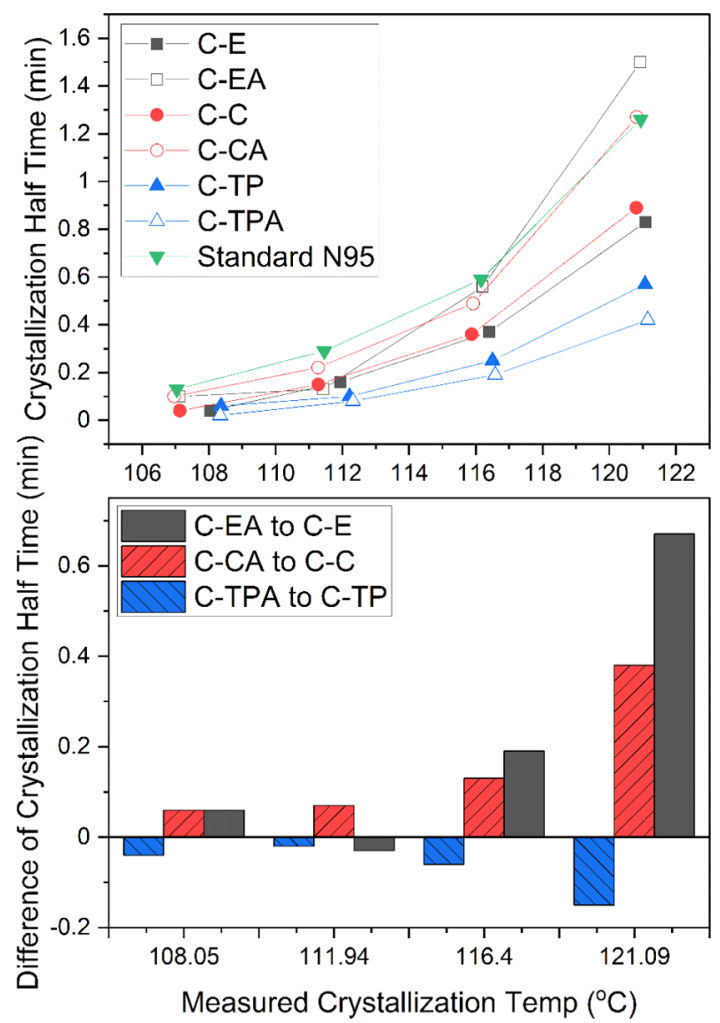

Figure 4. Isothermal DSC data plotting (top) time to crystallization peak vs isothermal crystallization temperature and (bottom) change in crystallization half time at each cool temperature upon the use of the additive.

observe that the TP samples have the fastest crystallization times. This is consistent with the vis-broken nature of the TP polymer system, which gives a lower molecular weight (and thus higher melt flow during melt blowing). Samples E and C show crystallization rates similar to that of the Standard N95 material. The additive increases the crystallization time for samples $\mathrm{C}$ and $\mathrm{E}$, while it decreases the crystallization time for sample TP (see the lower section of Figure 4). Of note, we see that longest crystallization times are (from the largest to smallest) C-EA, C-CA, Standard N95, C-C. Recall that the overall filtration performance is Standard N95 > C-CA > C-C, suggesting that slower crystallization rates are helpful in forming effective filter media.

Nonisothermal DSC gives the crystallization onset time and temperature for various cooling rates. Comparing the various melt-blown samples in Figure 5, we observe that the TP
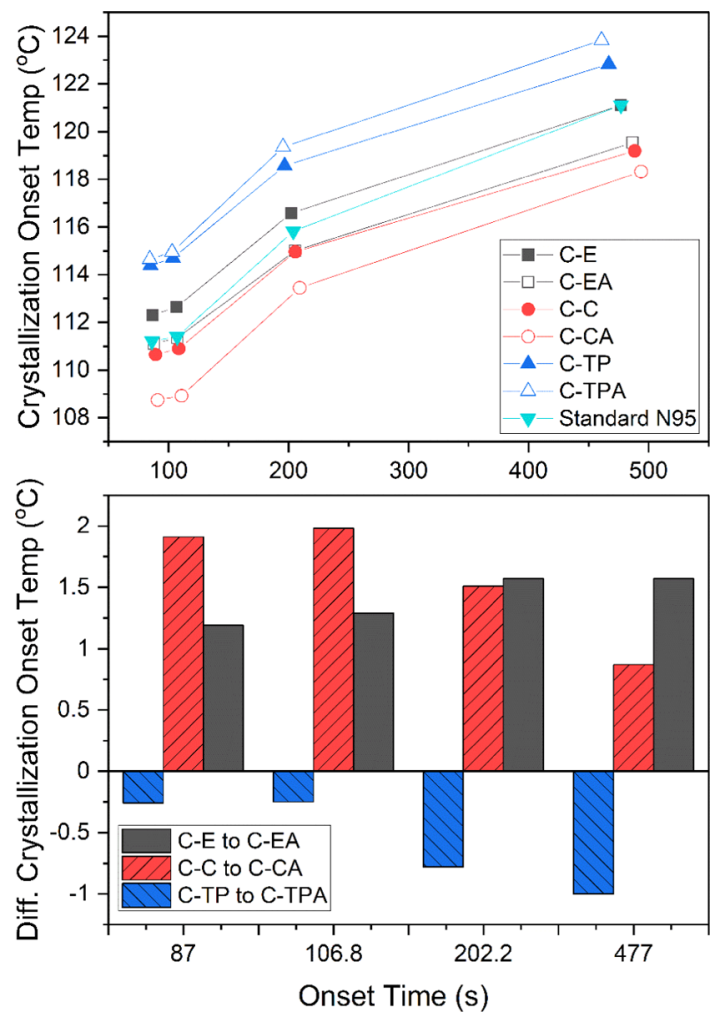

Figure 5. Nonisothermal DSC data plotting (top) crystallization onset temperature as a function of crystallization onset time and (bottom) the difference in onset temperature resulting from the additive. Note that materials were cooled at roughly $10,25,50$, and 60 ${ }^{\circ} \mathrm{C} / \mathrm{min}$ with crystallization onset times increasing with decreasing cooling rate.

systems have the highest crystallization onset temperatures. Like the isothermal DSC, the TP systems are likely outliers due to their low molecular weight. Sample E has very similar crystallization onset temperatures to the Standard N95 material and is slightly higher than sample $\mathrm{C}$. The additive decreases the crystallization onset temperature of samples while it increases the onset temperature of sample TP. Considering the crystallization onset temperature, we observe the highest onset temperatures, in decreasing order, as the TP systems, C-E, Standard N95, C-EA, C-C, C-CA. Momentarily disregarding the TP samples, we see that materials with high crystallization onset temperatures show good filtration performance. The TP samples and C-CA samples counter this trend but may be explained by their higher nucleation rates. The maximum cooling rates generated in DSC $\left(\approx 65^{\circ} \mathrm{C} /\right.$ $\mathrm{min})$ are not as fast those generated during the melt blowing process $\left(10^{3}-10^{4}{ }^{\circ} \mathrm{C} / \mathrm{s}\right)$, so it is possible that the faster cooling rates during melt blowing result in some variation compared to the crystallization onset temperatures observed by DSC.

The combined isothermal and nonisothermal DSC data suggest that a slow crystallization rate and a high crystallization onset temperature correlate with improved filtration performance in the charged systems. A slow crystallization rate and high crystallization onset temperature, combined with the nucleating effect of the additive, will help create a higher number of small crystallites. This crystalline-amorphous interface has been suggested as important in the development of electret formation and therefore filtration performance. $^{23-25,31}$ DSC analysis also included a determination of 
crystallinity. Table 2 compares the calculated initial crystallinity by DSC with that measured by X-ray diffraction (XRD).

Table 2. Initial Crystallinity as Measured by DSC Compared with XRD Crystallinity

\begin{tabular}{lcc}
\multicolumn{1}{c}{$\begin{array}{c}\text { sample } \\
\text { description }\end{array}$} & $\begin{array}{c}\text { initial \% crystallinity (DSC- } \\
\text { heating) }\end{array}$ & $\begin{array}{c}\text { crystallinity } \\
\text { (XRD) }\end{array}$ \\
NC-E & 44.4 & 50.8 \\
C-E & 42.5 & \\
C-EA & 43.2 & \\
NC-C & 42.7 & 41.9 \\
C-C & 42.7 & \\
C-CA & 42.3 & 47.7 \\
NC-TP & 46.6 & \\
C-TP & 46.6 & 46.5 \\
C-TPA & 44.7 & \\
Standard N95 & 43.1 & \\
E PP feedstock & 41.2 & \\
C PP feedstock & 36.3 & \\
TP PP feedstock & 43.4 & \\
additives & 31.0 &
\end{tabular}

The DSC and XRD results are in good agreement. All meltblown samples show crystallinity in the range of $42-47 \%$; this tight range is reasonable given the similarity of the materials and their processing conditions. There is no major trend between crystalline fraction and filtration performance.

3.4. Thermogravimetric Analysis. The samples generally show a perturbation around $160{ }^{\circ} \mathrm{C}$ (near the melting temperature of PP) followed by a major decomposition peak around $455{ }^{\circ} \mathrm{C}$. Run-to-run variation was observed to show a range of decomposition peaks from 458 to $446{ }^{\circ} \mathrm{C}$ in one sample. This suggests that minor differences between samples should not be overanalyzed. With this run-to-run variability taken into consideration, all samples show decomposition peak temperatures between 453 and $460{ }^{\circ} \mathrm{C}$, suggesting highly similar compositions. The residue was observed for all samples. For the pure PP feedstocks and pure PP melt-blown fibers, residue contents were generally low, between 0.2 and $0.5 \%$. The residue from the masterbatch additive was $1.9 \%$. The inclusion of the additive in the melt-blown fabrics increased the residue to around $1.5 \%$. The TP system showed higher residues in both the pure and modified samples, 0.5 and $2.25 \%$, respectively.

3.5. X-ray Photoelectron Spectroscopy. Eight samples were analyzed by XPS. Seven melt-blown fibers from this work (C-E, NC-EA, C-EA, C-C, NC-CA, C-CA, C-TP) and one standard N95 sample. The samples were prepared for analysis by cutting into $1 \mathrm{~cm} \times 1 \mathrm{~cm}$ pieces and attaching them to the sample holder with metal clips. All eight samples were analyzed together. An initial wide energy range survey spectrum was acquired to determine all elements present. Then, a set of narrow energy range core-level spectra were acquired for each identified element. In addition, the valence band region (0-30 eV binding energy) and the C KVV Auger spectrum were also acquired. Table S1 and Figure S11 compare the survey spectra and show the surface composition for each sample. All samples showed $>97$ atom $\% \mathrm{C}$ and small amounts of N, O, and $\mathrm{Si}$ (one sample showed a small amount of $\mathrm{Na}$ ). Samples C-E and C-C are expected to be pure polypropylene. Likewise, the stearatebased additive is expected to contribute some oxygen. There are no known sources of $\mathrm{N}, \mathrm{Si}$, or $\mathrm{Na}$. The oxygen content correlates with the application of charge. Corona treatment is a common industrial method of increasing the surface reactivity of a material by forming an oxygen-rich surface. The application of corona charging to develop the electret in the filter material also increased the surface oxygen content of the fibers. The C 1s, valence band, and C KVV spectra for the standard N95 sample were compared to the corresponding spectra taken from a published database for standard polypropylene (PP) in Figure S12. The C 1s was slightly wider than the database spectrum due primarily to the difference between a collection of fibers and a standard PP sheet material. The valence band and C KLL spectra for the N95 standard compare quite well to their database counterparts for PP. Figure S13 compares the C 1s and O 1s core-level spectra for the eight samples. It is clear that all of the samples show a similar single-peak $\mathrm{C} 1 \mathrm{~s}$ spectrum. Likewise, the $\mathrm{O} 1 \mathrm{~s}$ all show a single peak, centered at $\sim 531.5 \mathrm{eV}$ binding energy. This binding energy for $\mathrm{O}$ typically indicates $\mathrm{O}=\mathrm{C}$ bonding.

3.6. X-ray Diffraction. The PP beads and melt-blown fabrics were scanned with $\mathrm{Cu} \mathrm{K} \alpha$ radiation in a Panalytical $\mathrm{X}$ ray diffractometer in flat $\theta-2 \theta$ geometry to obtain diffraction patterns. All the beads and melt-blown fabrics exhibit predominantly single-phase monoclinic crystal structure $(\alpha$ polypropelene) with four dominant peaks as indicated in Figures 6 and S14. The slight noticeable difference in feedstock

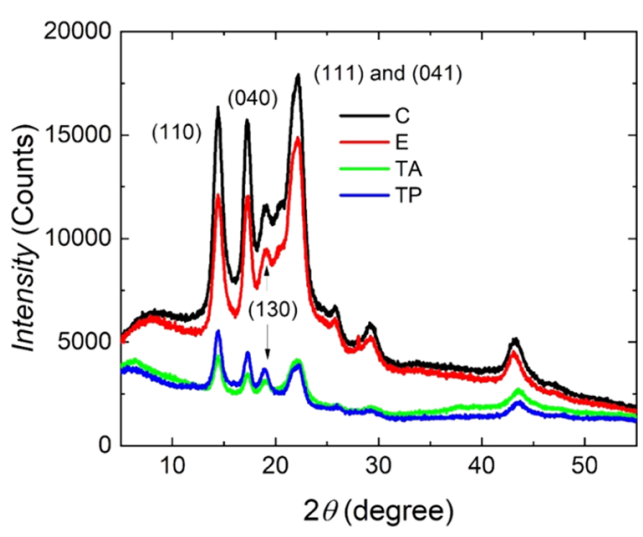

Figure 6. X-ray pattern for various sources of polypropylene beads, which show predominantly monoclinic crystal structure ( $\alpha$-form). In a clear look, the dominant peak for the $\mathrm{C}$ and $\mathrm{E}$ beads is combined (111)/(041) whereas Techmer and TP polymer beads exhibit dominant intensity at the (110) peak.

(Figure 6) and melt-blown fabrics (Figure S14) in the XRD patterns is the predominantly broadening of (110) peak, which suggests the variation of fiber diameter with fabrics axis being (110). The details of the variations among the feedstock's and melt-blown fabric patterns could be associated with processing condition-dependent lattice parameters of melt-blown fabrics since $\alpha$-PP homopolymer is reported to exhibit a big variation in lattice parameters $a, b, c$ and the deformed angle varied as $89.34 \leq \beta \leq 120.65$. $^{14}$

The variation in the surface charge of electret PP fibers generates varying crystallite sizes, e.g., their aggregates along with global strain in the fibers. It appears with charging; the fiber diameter is reduced slightly and hence increases the porosity. More detailed studies are being made to confirm this observation. These characteristics are manifested in the X-ray diffraction patterns in terms of peak width broadening. The crystallite sizes can be estimated from the X-ray patterns using 
Table 3. Comparison of FWHM, Crystallite Size, Crystallinity, and Strain in Beads and Melt-Blown Fibers

\begin{tabular}{|c|c|c|c|c|c|c|c|c|c|c|}
\hline \multirow[b]{3}{*}{$\begin{array}{l}\text { sample } \\
\text { fabrics }\end{array}$} & \multirow[b]{3}{*}{$\begin{array}{l}\text { crystal plane } \\
\quad(h k l)\end{array}$} & \multirow[b]{3}{*}{$\begin{array}{c}2 \theta \\
(\operatorname{deg})\end{array}$} & & & \multicolumn{2}{|c|}{ crystallite sizes } & \multirow[b]{3}{*}{$\begin{array}{c}D(\AA)= \\
\frac{k \lambda}{\mathrm{W}-\mathrm{H} \text { interept }}\end{array}$} & \multirow[b]{3}{*}{$\begin{array}{l}\text { crystallinity } \\
(\%)\end{array}$} & \multirow[b]{3}{*}{$\begin{array}{l}\text { DSC data } \\
\text { (melting) }\end{array}$} & \multirow[b]{3}{*}{$\begin{array}{c}\text { strain } \\
\text { (slope of a } \mathrm{W}-\mathrm{H} \text { plot) }\end{array}$} \\
\hline & & & \multicolumn{2}{|c|}{ FWHM $\left(\beta_{\mathrm{deg}}\right)$} & \multicolumn{2}{|c|}{$L_{h k l}=\frac{k \lambda}{\beta_{\mathrm{rad}} \cos (\theta)}$} & & & & \\
\hline & & & $\begin{array}{c}\text { Gaussian } \\
\text { fit (G) }\end{array}$ & $\begin{array}{c}\text { pseudo-Voigt } \\
1(\mathrm{PV})\end{array}$ & G & PV & & & & \\
\hline \multirow[t]{4}{*}{$\mathrm{C}-\mathrm{C}$} & 110 & 14.3 & 1.5 & 1.4 & 53.9 & 55.5 & 69.5 & 41.9 & $41.1 \%$ & 0.0077 \\
\hline & 040 & 16.8 & 2.1 & 1.5 & 38.4 & 53.8 & & 22.7 & & \\
\hline & 130 & 18.8 & 1.5 & 1.6 & 53.8 & 49.5 & & 16.6 & & \\
\hline & 111,041 & 21.6 & 1.9 & 1.9 & 40.8 & 43.3 & & 18.8 & & \\
\hline \multirow[t]{4}{*}{ C-E } & 110 & 14.0 & 1.7 & 1.2 & 48.3 & 66.9 & 21.6 & 50.8 & $44.0 \%$ & 0.0253 \\
\hline & 040 & 16.3 & 2.3 & 2.8 & 34.5 & 29.2 & & 18.1 & & \\
\hline & 130 & 18.5 & 1.3 & 1.7 & 63.9 & 46.2 & & 13.3 & & \\
\hline & 111,041 & 21.1 & 2.3 & 1.4 & 35.4 & 59.8 & & 14.0 & & \\
\hline \multirow[t]{4}{*}{ C-TP } & 110 & 14.3 & 1.9 & 1.9 & 42.3 & 42.3 & 28.3 & 47.7 & $45.0 \%$ & 0.0096 \\
\hline & 040 & 16.6 & 2.3 & 2.3 & 35.2 & 35.2 & & 26.1 & & \\
\hline & 130 & 18.8 & 1.9 & 1.9 & 42.7 & 42.7 & & 9.9 & & \\
\hline & 111,041 & 21.5 & 2.2 & 2.2 & 37.6 & 37.6 & & 16.3 & & \\
\hline \multirow{4}{*}{$\begin{array}{l}\text { standard } \\
\text { N95 }\end{array}$} & 110 & 14.4 & 1.9 & 1.4 & 43.3 & 57.0 & 57.3 & 46.5 & $42.6 \%$ & 0.0075 \\
\hline & 040 & 16.6 & 2.3 & 2.7 & 35.3 & 29.5 & & 19.0 & & \\
\hline & 130 & 17.8 & 1.4 & 1.7 & 56.3 & 46.7 & & 14.3 & & \\
\hline & 111,041 & 21.6 & 2.2 & 1.5 & 37.5 & 52.6 & & 20.2 & & \\
\hline \multirow[t]{4}{*}{ C-CA } & 110 & 14.3 & 2.1 & 2.1 & 37.8 & 37.8 & 40.6 & 49.1 & & 0.0004 \\
\hline & 040 & 16.5 & 2.0 & 2.4 & 41.2 & 34.1 & & 21.1 & & \\
\hline & 130 & 18.6 & 1.8 & 1.4 & 44.5 & 57.0 & & 10.7 & & \\
\hline & 111,041 & 21.3 & 2.2 & 2.2 & 36.4 & 36.8 & & 15.2 & & \\
\hline \multirow[t]{4}{*}{ C-EA } & 110 & 14.3 & 2.5 & 2.0 & 31.8 & 40.8 & 31.4 & 48.0 & & 0.0074 \\
\hline & 040 & 16.6 & 1.8 & 2.2 & 44.3 & 37.3 & & 16.7 & & \\
\hline & 130 & 18.4 & 1.8 & 2.5 & 45.9 & 31.9 & & 12.3 & & \\
\hline & 111,041 & 21.0 & 2.4 & 2.1 & 33.8 & 37.8 & & 19.0 & & \\
\hline \multirow[t]{4}{*}{ С-TPA } & 110 & 14.2 & 1.7 & 1.4 & 46.3 & 58.9 & 37.2 & 41.5 & & 0.0343 \\
\hline & 040 & 16.4 & 2.4 & 2.7 & 33.7 & 30.0 & & 19.9 & & \\
\hline & 130 & 18.6 & 1.2 & 1.7 & 69.5 & 47.5 & & 10.0 & & \\
\hline & 111,041 & 21.3 & 3.0 & 1.9 & 27.4 & 42.3 & & 18.6 & & \\
\hline \multirow[t]{4}{*}{ C Beads } & 110 & 14.4 & 1.02 & 0.30 & 78.77 & 265.68 & & 18.13 & & \\
\hline & 40 & 17.3 & 0.67 & 0.24 & 120.55 & 333.65 & & 0.73 & & \\
\hline & 130 & 20.1 & 7.37 & 4.14 & 10.95 & 19.48 & & 15.22 & & \\
\hline & $111 / 041$ & 22.1 & 1.29 & 1.56 & 62.76 & 51.72 & & 60.78 & & \\
\hline
\end{tabular}

the Scherrer equation, whereas the strain in the crystallites is estimated using the Williamson-Hall plot or the WarrenAverbach method. In Warren-Averbach method, the X-ray diffraction pattern is expected to have multiple peaks of specific family of plane diffraction such as $[h 00],[0 k 0]$, or [00l]. In PP fibers, only first-order peaks of various diffraction planes were identified, as indicated in Figure S15, so the $\mathrm{W}-\mathrm{H}$ plot becomes more relevant to estimate the crystallite sizes and global strain. Instead of fitting the complex mathematical curves that are difficult to deconvolute the information, we used the OriginLab peak analyzer tool that allows the removal of instrumental background generating the user-defined baseline fit as shown in Figure S15. The peak analyzer tool allows the user to select the peak positions and background as well as tolerance management to specify whether to count a peak in crystallinity analysis or not. All the peaks with relative height less than $20 \%$ were not included in the study as shown in broad low-height peaks. The inset shows the peak width separated by blue vertical lines and peak positions marked with numbered red lines. The crystallinity percent was estimated as the ratio of the individual peak area to the total area under the curve, i.e.,

$$
\text { crystallinity }(\%)=\frac{\text { area of selected peak }}{\text { area under the curve }} \times 100 \%
$$

Unimodal isotropic powder provides ideally Gaussian peaks in the XRD pattern. As crystallite sizes and global strain in fibers vary, the XRD pattern deviates from unimodal Gaussian distribution. Here, melt-blown fabrics sheets are colis and rolls of PP fibers, which may make the XRD patterns deviate from ideal Gaussian patterns. The background for the fit was estimated with the empty scan of the fabric holder for the experiment. The shape was consistently maintained for all the fittings. We determined the full width at half-maximum (fwhm) of the various melt-blown fabrics from the XRD patterns as indicated from Gaussian and pseudo-Voigt peak fits (as shown in Figure S16 for melt-blown C-C) and estimated the crystallite size $\left(L_{h k l}\right)$ using Scherrer's equation.

$$
L_{h k l}=\frac{k \lambda}{\beta \cos (\theta)}
$$

where $\lambda=1.54056 \AA$ is the $\mathrm{Cu} \mathrm{K} \alpha$ radiation wavelength, $\beta$ is the full width at half-maxima of the peak at position $2 \theta$, and $k$ is a geometry-dependent structural factor $(k=0.9$ for $\mathrm{pp}$ fabrics). ${ }^{25}$ The estimated crystallite sizes are greatly varying 
among the literature. ${ }^{12,14 b, 29}$ The crystallite sizes of the beads are in general found to be larger than melt-blown fibers. Thus, the smaller crystallite size formation is associated with the melt-blown processing technique, which improves the electret formation and therefore the filtration efficiency of the fabrics. The crystallinity \% of the C-E polymer fabrics comes highest among the investigated fabrics. Interestingly, some of the fiber pictures in C-E fabric SEM images shown in Figures 2 and S4 look larger in diameter with irregular shape; large crystallite size is probably playing a role in such morphology variation. To investigate the role of global strain in the observed crystallite broadening in melt-blown fabrics, a $\mathrm{W}-\mathrm{H}$ plot was prepared using the peak center and FWHM in Table 3 as

$$
\beta \cos (\theta)=\frac{k \lambda}{D}+4 \epsilon \sin (\theta)
$$

where $\beta_{\text {rad }}$ is the total width, $k=0.9$ is the Scherrer constant, $\lambda$ $=1.54056 \AA$ is $\mathrm{Cu} \mathrm{K} \alpha$ radiation, $D=$ crystallite size, and $\epsilon$ is strain. $^{32}$ A typical $\mathrm{W}-\mathrm{H}$ plot for $\mathrm{C}-\mathrm{C}$ melt-blown fiber is presented in Figure S17. The $Y$-intercept provides the equation for crystallite size as

$$
D=\frac{k \lambda}{Y \text {-intercept }}
$$

The smaller the $Y$-intercept, the larger the crystallite size and vice versa. Similarly, the positive slope $\epsilon$ represents the fibers under extension conditions under electrostatic stress of the surface charge of the electret and vice versa. The crystallite sizes obtained from a $\mathrm{W}-\mathrm{H}$ plot are comparable to Scherrer's equation except in $\mathrm{C}-\mathrm{C}$ fabrics, whereas the predictable relation for the global stress was not detected among the fabrics. The rest of the samples are predominantly alike to the selective four samples above in terms of major diffraction peaks understudy as shown in Figure S18, which would not be enough evidence to extract any significantly different information among them.

3.7. Neutron Scattering. Inelastic neutron scattering (INS) is a powerful tool to study molecular vibration. Compared to other spectroscopic methods such as IR and Raman, INS has several advantages, one of which is its sensitivity to low-frequency vibrational modes. In this case, these low-frequency modes correspond to the translational and rotational motion of the polymer chains, which may reflect the local arrangement of the molecules such as degree of ordering, packing density, and crystallinity. INS was used to characterize and compare the samples at the VISION beamline of the Spallation Neutron Source. The measured spectra are almost identical at the higher energy range, which is not surprising given that these samples are essentially all polypropylene and the high-frequency intramolecular motions are not affected by the subtle differences at intermolecular and longer length scales. However, in the low-frequency (energy) range between 2 and $10 \mathrm{meV}$, systematic differences are indeed observed, as shown in Figure S19. The broad peak in this energy range generally corresponds to the motion of the polymer chains relative to each other, such as translation and rotation. Based on our density functional theory simulation of the vibrational dynamics, the translational motion has relatively low frequencies compared to the rotational motion. Therefore, the lower-frequency part of the peak is mainly contributed by translational motion, and the higher frequency part is mainly due to the rotational motion. For a specific motion, the INS peak intensity scales with the amplitude of the molecular vibration. A general trend observed in Figure S19 is that the rotational peak intensity is relatively strong in samples with no additives, and the additives seem to enhance the translational peak intensity, changing the shape of the peak. This same trend is true for all the three groups of samples (E, C, TP). This indicates that the additives cause a larger amplitude of motion for the translational modes, which could have its structural origin in more structural disorder and smaller crystallite (e.g., more crystalline-amorphous interface).

3.8. FTIR Analysis of Tacticity. Fourier transform infrared (FTIR) spectroscopy was collected over the range 2000-600 $\mathrm{cm}^{-1}$ to characterize tacticity. Using a linear baseline between approximately 1056 and $927 \mathrm{~cm}^{-1}$, the ratios of the 997 and 972 peaks were calculated for all samples and used to determine the $\%(\mathrm{~mm})$ isotacticity according to ref 33 .

$$
\frac{A_{997}}{A_{972}}=1.08(\mathrm{~mm})-0.1
$$

All samples showed a high degree of isotacticity of around $86 \%$ (high of $88 \%$, low of $83 \%$ ). There is no trend in the degree of tacticity between suppliers and samples implying that the variation is experimental, not real. Supplier provided information for one sample suggests that isotacticity levels are higher, near $99 \%$. The FTIR data can thus be interpreted to conclude that there are no major differences in tacticity between samples.

\section{CONCLUSIONS}

Melt-blown polypropylene fabrics were manufactured by melt blowing three commercial grades of high melt flow polypropylene and a stearate-based polypropylene masterbatch additive. The resulting fabrics were corona treated by a custom system to generate electrets and demonstrated vastly improved filtration efficiencies. The fabrics were characterized to understand their performance and differences. Despite the nearly identical feedstocks, the materials performed very differently. From the analysis performed here, the most differentiating factor appears to be related to crystallization. Materials with higher crystallization onset temperatures, slower crystallization rates, and larger number of crystallites are more effective at filtration. The stearate-based additive improves the filtration performance by decreasing crystallite size to a point, but for polymers with sufficient nucleation and thus small enough crystallite size, the additive has no further effect. In the case of the vis-breaking TP samples, the additive decreases performance. The effect of crystallization on filtration supports the fact that charge trapping at the crystalline-amorphous interface improves the electret formation and therefore improves electrostatic filtration effectiveness.

\section{EXPERIMENTAL SECTION}

5.1. Filtration Efficiency. Filtration efficiency was characterized by measuring the penetration of the filter media by a sodium chloride aerosol particle generator according to the standard testing media. Two layers of the melt-blown filter media were stacked together for testing. The airflow rate during the test was $50 \mathrm{lpm}$.

5.2. SEM. A scanning electron microscope (SEM) was conducted to assess the morphology of the melt-blown fibers and characterize the fiber diameters. The SEM images were obtained in a Carl Zeiss Merlin SEM operating at $1 \mathrm{kV}$. Both the raw materials and the finished melt-blown fibers were analyzed. 
5.3. XPS. X-ray photoelectron spectroscopy (XPS) was performed using a Thermo Scientific (Waltham, MA) Model K-Alpha XPS instrument. The instrument utilizes monochromated, microfocused, $\mathrm{Al} \mathrm{K \alpha}$ X-rays $(1486.6 \mathrm{eV}$ ) with a variable spot size (i.e., $30-400 \mu \mathrm{m})$. Analyses of the samples were all performed with the $400 \mu \mathrm{m}$ X-ray spot size for maximum signal and to obtain an average surface composition over the largest possible area. The instrument has a hemispherical electron energy analyzer equipped with a 128-channel detector system. Base pressure in the analysis chamber is typically $2 \times$ $10^{-9}$ mbar or lower. Samples were mounted to the sample holder using metal clips. Survey spectra (pass energy $=200 \mathrm{eV}$ ) were acquired for qualitative and quantitative analysis, and high-resolution core-level spectra (pass energy $=50 \mathrm{eV}$ ) were acquired for detailed chemical state analysis. All spectra were acquired with the charge neutralization flood gun turned on to maintain a stable analysis condition. The flood gun uses a combination of low-energy electrons and argon ions for optimum charge compensation. The typical pressure in the analysis chamber with the flood gun operating is $2 \times$ $10^{-7}$ mbar. Data were collected and processed using the Thermo Scientific Avantage XPS software package (v.5.96). Peak fitting was performed using mixed Gaussian/Lorentzian peak shapes and a Shirley/Smart type background.

5.4. XRD. A panalytic $\mathrm{X}$-ray diffractometer with $\mathrm{Cu} \mathrm{K} \alpha$ radiation in a flat $\theta-2 \theta$ geometry was used to obtain diffraction patterns on $\mathrm{PP}$ melts and fabrics. XRD data was used to determine the degree of crystallinity and the crystallite size of PP material.

5.5. DSC. DSC was used to measure melting temperatures, crystallization rates, and degree of crystallinity. The instrument utilized was a TA Instruments Q100 DSC. As noted above, the melt blowing process results in fast cooling rates. Therefore, isothermal DSC was conducted to compare general material properties and nonisothermal DSC to compare differences in the material during fast cooling rates more indicative of the melt blowing process. Specifically, the material was cooled at $10,25,50$, and $60{ }^{\circ} \mathrm{C} / \mathrm{min}$ in the nonisothermal tests with the time to crystallization onset and crystallization onset temperature reported. Crystallinity was determined according to the First Law method. ${ }^{34}$ Briefly, the First Law method evaluates the residual enthalpy of the sample at the lower temperature $T_{1}$ (room temp or above but close to $T_{\mathrm{g}}$ ) and determines the initial degree of crystallinity of the sample prior to heating. This method is more accurate than commonly used approaches (linear baselines, enthalpy of fusion at $T_{\mathrm{m}}$ ) because the enthalpy of fusion is measured at the lower temperature and correction is made for the specific heat changes. Samples were heated at $100{ }^{\circ} \mathrm{C} / \mathrm{min}$ in the isothermal and nonisothermal tests and at $10{ }^{\circ} \mathrm{C} / \mathrm{min}$ for the initial crystallinity tests. The melting enthalpy for $100 \%$ crystalline PP was taken as $207.1 \mathrm{~J} / \mathrm{g}$. All tests were run under flowing nitrogen.

5.6. TGA. Thermogravimetric analysis (TGA) was run on a TA Instruments Q600 SDT dual beam horizontal balance. Samples were taken from melt-blown fabric as well as the PP as provided; sizes were approximately $10 \mathrm{mg}$. Samples were heated from room temperature to $600{ }^{\circ} \mathrm{C}$ at $10{ }^{\circ} \mathrm{C} / \mathrm{min}$ under flowing nitrogen.

5.7. INS. Inelastic neutron scattering (INS) experiments were performed at Beamline 16B (VISION), Spallation Neutron Source, Oak Ridge National Laboratory. VISION is an indirect-geometry neutron spectrometer with a broad dynamic range ( -2 to $1000 \mathrm{meV})$ and high resolution $(\sim 1.5 \%$ of energy transfer $)$. INS spectra were collected at $5 \mathrm{~K}$ to minimize the thermal motion of atoms that suppresses the signal intensities. The samples were clamped between borated (neutron-absorbing) aluminum frames, and slits were adjusted to illuminate only the polypropylene films. No background subtraction was needed. To compare the spectra from different samples on the same basis, each spectrum was further normalized by its total integrated intensity (which scales with the amount of the sample in the neutron beam).

5.8. FTIR. FTIR was collected over the range $2000-600 \mathrm{~cm}^{-1}$ to characterize tacticity. Using a linear baseline between approximately 1056 and $927 \mathrm{~cm}^{-1}$, the ratio of the 997 and 972 peaks were calculated for all samples and used to determine the \%(mm) isotacticty according to ref 33 .

\section{ASSOCIATED CONTENT}

\section{(s) Supporting Information}

The Supporting Information is available free of charge at https://pubs.acs.org/doi/10.1021/acsapm.0c01294.

Melt-blown process schematics; polymer feedstock, fabrication process, and characterizations; and resistance data, SEM, XPS, XRD, and INS figures (PDF)

\section{AUTHOR INFORMATION}

\section{Corresponding Authors}

Gregory S. Larsen - Oak Ridge National Laboratory, Oak Ridge, Tennessee 37831, United States; Email: larsengs@ ornl.gov

Merlin Theodore - Oak Ridge National Laboratory, Oak Ridge, Tennessee 37831, United States; Email: theodorem@ ornl.gov

Mariappan Parans Paranthaman - Oak Ridge National Laboratory, Oak Ridge, Tennessee 37831, United States; (1) orcid.org/0000-0003-3009-8531; Email: paranthamanm@ornl.gov

\section{Authors}

Yongqiang Cheng - Oak Ridge National Laboratory, Oak Ridge, Tennessee 37831, United States; (1) orcid.org/00000002-3263-4812

Luke L. Daemen - Oak Ridge National Laboratory, Oak Ridge, Tennessee 37831, United States

Tej N. Lamichhane - Oak Ridge National Laboratory, Oak Ridge, Tennessee 37831, United States

Dale K. Hensley - Oak Ridge National Laboratory, Oak Ridge, Tennessee 37831, United States

Kunlun Hong - Oak Ridge National Laboratory, Oak Ridge, Tennessee 37831, United States; 이 orcid.org/0000-00022852-5111

Harry M. Meyer, III - Oak Ridge National Laboratory, Oak Ridge, Tennessee 37831, United States

Steven J. Monaco - RJ Lee Group, Monroeville, Pennsylvania 15146, United States

Alan M. Levine - RJ Lee Group, Monroeville, Pennsylvania 15146, United States

Richard J. Lee - RJ Lee Group, Monroeville, Pennsylvania 15146, United States

Emma Betters - Oak Ridge National Laboratory, Oak Ridge, Tennessee 37831, United States

Kim Sitzlar - Oak Ridge National Laboratory, Oak Ridge, Tennessee 37831, United States

Jesse Heineman - Oak Ridge National Laboratory, Oak Ridge, Tennessee 37831, United States

Justin West - Oak Ridge National Laboratory, Oak Ridge, Tennessee 37831, United States

Peter Lloyd - Oak Ridge National Laboratory, Oak Ridge, Tennessee 37831, United States

Vlastimil Kunc - Oak Ridge National Laboratory, Oak Ridge, Tennessee 37831, United States

Lonnie Love - Oak Ridge National Laboratory, Oak Ridge, Tennessee 37831, United States

Complete contact information is available at:

https://pubs.acs.org/10.1021/acsapm.0c01294

\section{Notes}

The authors declare no competing financial interest. 


\section{ACKNOWLEDGMENTS}

This research was supported by the DOE Office of Science through the National Virtual Biotechnology Laboratory (NVBL), a consortium of DOE national laboratories focused on response to COVID-19, with funding provided by the Coronavirus CARES Act. SEM microstructural characterizations and FTIR measurements were conducted at the Center for Nanophase Materials Sciences, which is the US Department of Energy Office of Science User Facility. Neutron scattering experiment was performed at ORNL's Spallation Neutron Source, supported by the Scientific User Facilities Division, Office of Basic Energy Sciences, US DOE, under contract no. DE-AC0500OR22725 with UT Battelle, LLC. Thanks to Dr. Peter Tsai for helpful discussions. The US Government retains and the publisher, by accepting the article for publication, acknowledges that the US government retains a nonexclusive, paid-up, irrevocable, worldwide license to publish or reproduce the published form of this manuscript, or allow others to do so, for US Government purposes. DOE will provide public access to these results of federally sponsored research in accordance with the DOE Public Access Plan (http://energy.gov/downloads/doe-public-access-plan).

\section{REFERENCES}

(1) Viscusi, D. J.; Bergman, M. S.; Eimer, B. C.; Shaffer, R. E. Evaluation of five decontamination methods for filtering facepiece respirators. Ann. Occup. Hyg. 2009, 53, 815-827.

(2) Lin, T. H.; Chen, C. C.; Huang, S. H.; Kuo, C. W.; Lai, C. Y.; Lin, W. Y. Filter quality of electret masks in filtering $14.6-594 \mathrm{~nm}$ aerosol particles: Effects of five decontamination methods. PLoS One 2017, 12, No. e0186217.

(3) Ullah, S.; Ullah, A.; Lee, J.; Jeong, Y.; Hashmi, M.; Zhu, C.; Il Joo, K.; Cha, H. J.; Kim, I. S. Reusability Comparison of Melt-Blown vs Nanofiber Face Mask Filters for Use in the Coronavirus Pandemic. ACS Appl. Nano Mater. 2020, 3, 7231-7241.

(4) Bartoszko, J. J.; Farooqi, M. A. M.; Alhazzani, W.; Loeb, M. Medical masks vs N95 respirators for preventing COVID-19 in healthcare workers: A systematic review and meta-analysis of randomized trials. Influenza Other Respir. Viruses 2020, 14, 365-373.

(5) Richter, W.; Hofacre, K.; Willenberg, Z. Final Report for the Bioquell Hydrogen Peroxide Vapor (HPV) Decontamination for Reuse of N95 Respirators; FDA, Battelle, Contract No. HHSF223201400098C, 2016.

(6) Hinton, D. M. FDA EUA for Battelle CCDS, 2020. https:// www.battelle.org/inb/battelle-critical-care-decontamination-systemfor-covid19.

(7) Drabek, J.; Zatloukal, M. Meltblown technology for production of polymeric microfibers/nanofibers: A review. Phys. Fluids 2019, 31, No. 091301

(8) Dutton, K. C. Overview and Analysis of the Meltblown Process and Parameters. J. Text. Apparel Technol. Manage. 2008, 6, No. 2008.

(9) Bresee, R. R.; Ko, W.-C. Fiber Formation during Melt Blowing. Int. Nonwovens J. 2018, os-12, 7.

(10) Hassan, M. A.; Yeom, B. Y.; Wilkie, A.; Pourdeyhimi, B.; Khan, S. A. Fabrication of nanofiber meltblown membranes and their filtration properties. J. Membr. Sci. 2013, 427, 336-344.

(11) Drabek, J.; Zatloukal, M. Influence of long chain branching on fiber diameter distribution for polypropylene nonwovens produced by melt blown process. J. Rheol. 2019, 63, 519-532.

(12) Shambaugh, B. R.; Papavassiliou, D. V.; Shambaugh, R. L. Modifying Air Fields To Improve Melt Blowing. Ind. Eng. Chem. Res. 2012, 51, 3472-3482.

(13) Jarecki, L.; Ziabicki, A.; Lewandowski, Z.; Blim, A. Dynamics of air drawing in the melt blowing of nonwovens from isotactic polypropylene by computer modeling. J. Appl. Polym. Sci. 2011, 119, $53-65$.
(14) (a) Qin, X. H.; Wang, S. Y. Filtration properties of electrospinning nanofibers. J. Appl. Polym. Sci. 2006, 102, 12851290. (b) Wang, J.; Dou, Q.; Chen, X.; Li, D. Crystal structure and morphologies of polypropylene homopolymer and propylene-ethylene random copolymer: Effect of the substituted 1,3,5-benzenetrisamides. J. Polym. Sci., Part B: Polym. Phys. 2008, 46, 1067-1078.

(15) Kilic, A.; Shim, E.; Pourdeyhimi, B. Electrostatic Capture Efficiency Enhancement of Polypropylene Electret Filters with Barium Titanate. Aerosol Sci. Technol. 2015, 49, 666-673.

(16) Zhou, Z. J.; Zhou, B.; Tseng, C. H.; Hu, S. C.; Shiue, A.; Leggett, G. Evaluation of characterization and filtration performance of air cleaner materials. Int. J. Environ. Sci. Technol. 2020, 1-12.

(17) Barrett, L. W.; Rousseau, A. D. Aerosol Loading Performance of Electret Filter Media. Am. Ind. Hyg. Assoc. J. 2010, 59, 532-539.

(18) Park, H.-S.; Park, Y. O. Filtration properties of electrospun ultrafine fiber webs. Korean J. Chem. Eng. 2005, 22, 165-172.

(19) Tsai, P. P.; Schreuder-Gibson, H.; Gibson, P. Different electrostatic methods for making electret filters. J. Electrost. 2002, 54, 333-341.

(20) Tsai, P. P.; Wadsworth, L. C. Method and Apparatus for the Electrostatic Charging of a Web or Film. U.S. Patent US5,401,4461995.

(21) Tsai, P. P.-y.; Qin, G.-w.; Hassenboehler, C. Comparison of Electrostatic Charging at Different Locations in the Melt Blowing Process. Int. Nonwovens J. 2000, os-9, No. 1558925000OS-90.

(22) Sessler, G. M. Electrets: recent developments. J. Electrost. 2001, 51-52, 137-145.

(23) Zhang, H. F.; Liu, N.; Zeng, Q. R.; Liu, J. X.; Zhang, X.; Ge, M. Z.; Zhang, W.; Li, S. Y.; Fu, Y. J.; Zhang, Y. Design of Polypropylene Electret Melt Blown Nonwovens with Superior Filtration Efficiency Stability through Thermally Stimulated Charging. Polymers 2020, 12, No. 2341.

(24) Zhang, H.; Liu, J.; Zhang, X.; Huang, C.; Jin, X. Design of electret polypropylene melt blown air filtration material containing nucleating agent for effective PM2.5 capture. RSC Adv. 2018, 8, 7932-7941.

(25) Xiao, H. M.; Gui, J. Y.; Chen, G. J.; Xiao, C. P. Study on correlation of filtration performance and charge behavior and crystalline structure for melt-blown polypropylene electret fabrics. J. Appl. Polym. Sci. 2015, 132, No. 42807.

(26) Mohmeyer, N.; Behrendt, N.; Zhang, X.; Smith, P.; Altstädt, V.; Sessler, G. M.; Schmidt, H.-W. Additives to improve the electret properties of isotactic polypropylene. Polymer 2007, 48, 1612-1619.

(27) Busico, V.; Cipullo, R. Microstructure of polypropylene. Prog. Polym. Sci. 2001, 26, 443-533.

(28) van der Burgt, F.P. Crystallization of Isotactic Polypropylene: The Influence of Stereo-Defects; Technische Universiteit Eindhoven: Eindhoven, 2002

(29) Shamiri, A.; Chakrabarti, M. H.; Jahan, S.; Hussain, M. A.; Kaminsky, W.; Aravind, P. V.; Yehye, W. A. The Influence of ZieglerNatta and Metallocene Catalysts on Polyolefin Structure, Properties, and Processing Ability. Materials 2014, 7, 5069-5108.

(30) Gómez-Elvira, J. M.; Tiemblo, P.; Elvira, M.; Matisova-Rychla, L.; Rychly, J. Relaxations and thermal stability of low molecular weight predominantly isotactic metallocene and Ziegler-Natta polypropylene. Polym. Degrad. Stab. 2004, 85, 873-882.

(31) Guliakova, A. A.; Galikhanov, M. F.; Galeeva, L. R.; Gilfanova, S. V.; Fang, P. Investigation of electret and filtering properties of polypropylene-based nonwoven fabrics and its composites with 2 vol $\%$ of silicon dioxide inclusions. IEEE Trans. Dielectr. Electr. Insul. 2020, 27, 1656-1661.

(32) Kim, Y. Y.; Schenk, A. S.; Ihli, J.; Kulak, A. N.; Hetherington, N. B.; Tang, C. C.; Schmahl, W. W.; Griesshaber, E.; Hyett, G.; Meldrum, F. C. A critical analysis of calcium carbonate mesocrystals. Nat. Commun. 2014, 5, No. 4341.

(33) Burfield, D. R.; Loi, P. S. T. The use of infrared spectroscopy for determination of polypropylene stereoregularity. J. Appl. Polym. Sci. 1988, 36, 279-293. 
(34) Kong, Y.; Hay, J. N. The measurement of the crystallinity of polymers by DSC. Polymer 2002, 43, 3873-3878. 